\title{
Achieved availability importance measure for enhancing reliability- centered maintenance decisions
}

Proc IMechE Part O:

$\mathrm{J}$ Risk and Reliability

20I5, Vol. 229(I) 62-72

(c) IMechE 2014

Reprints and permissions:

sagepub.co.uk/journalsPermissions.nav DOI: $10.1177 / 1748006$ X 14550849

pio.sagepub.com

(SAGE

\author{
Mary A Gravette' and Kash Barker²
}

\begin{abstract}
An effective defense strategy requires aircraft, among other weapons systems, to be available and ready for use when circumstances deem necessary. This article offers a set of importance measures to identify the critical components in a system from their influence on system-achieved availability, a common Department of Defense availability calculation that is a ratio of mean time between maintenance and total system time, including mean maintenance time. With these measures, more effective maintenance plans, including inspection and supply inventory, can focus on those components that more significantly impact achieved availability. A decision-making formulation results from these component importance measures, and an example based on a US Air Force system illustrates the modeling contributions.
\end{abstract}

\section{Keywords}

Achieved availability, reliability-centered maintenance, component importance measure, inspections

Date received: 12 May 20I4; accepted: 18 August 2014

\section{Introduction and motivation}

The Department of Defense (DoD) ${ }^{1}$ uses three main metrics to measure the quality of one of its systems: reliability, maintainability, and availability. Particularly within the US Air Force, high-quality aircraft equipment requires high-performance values for all three metrics: reliable (ability to last as long as intended) and maintainable (ability to be fixed with minimum effort and time) to make the aircraft equipment available (accessible when needed). Availability, or the probability that a system is performing its required function at a given point in time when operated and maintained in a prescribed manner, ${ }^{2}$ is perhaps the key metric of the three.

The availability of DoD systems is threatened by obsolescence. For example in the US Air Force, the cost to replace over $500 \mathrm{KC}-135 \mathrm{~s}$, which debuted in the mid-1950s, has been estimated in the tens of billions of dollars with a replacement plan lasting for several decades. $^{3}$ A budget reduction of about $29 \%$ since 1990 has "forced the branches of the military to extend the life of current legacy systems with significant reductions in new acquisitions of replacement systems." 4 Such new purchases are usually restricted due to funding limitations, making redesigning or generating redundancy for improved reliability not an option. ${ }^{5,6}$ As such, the only remaining option to improve system availability is to enhance the maintenance methods during sustainment of the system. This leads to the need for an optimal maintenance policy to have the maximum positive impact on availability. ${ }^{7,8}$

Recent DoD focus has been directed toward improving the decision-making process for system sustainment, including maintenance, repair, and overhaul (MRO) operations and the acquisition of MRO parts. MRO depot resources must be dynamically assigned to reflect changes in priority driven by critical supply needs and internal parts' shortages with the goal of reducing cost and lead times, meeting due dates, and maximizing availability of DoD weapon systems. Streamlined MRO activities, including the scheduling of system maintenance, the acquisition of parts (e.g. spare part shortages have been a concern in the $\mathrm{DoD}^{9}$ ), and the optimal performance of supply chain

\footnotetext{
'Tinker Air Force Base, Oklahoma City, OK, USA

${ }^{2}$ School of Industrial and Systems Engineering, University of Oklahoma, Norman, OK, USA

\section{Corresponding author:}

Kash Barker, School of Industrial and Systems Engineering, University of Oklahoma, 202 W Boyd St, Rm. 124, Norman, OK 73019, USA.

Email: kashbarker@ou.edu
} 
operations, is key to keeping these aging systems available.

One means to tighten MRO costs is to focus on a primary set of components that most affect system performance. The analysis of systems, regardless of domain, often includes determining which system components are most influential on the performance of the system. Given the context above, the task of detecting the system, subsystem, or component on which to focus efforts (e.g. MRO activities) to gain the most improvement (e.g. improved availability) for the least cost is an important one. Component importance measures (CIMs), a well-studied topic in reliability engineering, ${ }^{10}$ measure the influence of particular components on the reliability of an overall system.

In a couple of recent theoretical exercises, importance measures have been developed to focus on availability metrics to determine the component in a system that most influences overall system availability. ${ }^{11,12}$ This article extends this recent work by (1) analyzing availability-based component importance for some specific reliability and maintainability metrics that comprise achieved availability, an important DoD metric and (2) proposing a simple decision-making formulation to highlight how inspections can impact availability for those components found to be most impactful to the system. Subsequent sections of this paper provide methodological background to the availability-based importance measure for achieved availability, a DoD-inspired illustrative example, and concluding remarks.

\section{Background and literature review}

Advancing technology and tighter budgets have prompted the desire to avoid failures before they occur, broadly referred to as preventive maintenance (PM). There remained a desire to do more for lessmore availability and reliability with focus on safety and environmental impacts while keeping budgets to a minimum - ultimately leading to the philosophy of reliability-centered maintenance (RCM). ${ }^{13}$ Those who plan on using RCM do so because they expect to gain longer availability times, lower costs, and better control and decisions. ${ }^{14}$ With the idea of RCM in mind, we review background on calculations for component and system availability, as well as importance measures.

\section{Availability classification and quantification}

The reliability, availability, and maintainability performance metrics of a system have fundamental relationships. Given our interests in $\mathrm{DoD}^{1}$ weapons systems in particular, we examine the similarity and differences among the DoD definitions of these system performance metrics:
- Reliability is the probability that an item can perform its intended function(s) without failure for a specified time under stated conditions.

- Availability is a measure of the degree to which an item is in an operable state and can be committed at the start of a mission when the mission is called for at an unknown (random) point in time.

- Maintainability is the probability that an item can be retained in, or restored to, a specified condition in a given time when maintenance is performed by personnel having specified skill levels, using prescribed procedures and resources, at each prescribed level of maintenance and repair.

Reliability is a metric that is often optimized during a system's design phase, where system configurations and component redundancies are considered to maximize system reliability or related mean time to failure metrics. However, after the design phase is completed, there is often no further action possible with the existing components to improve their reliability. As reliability as a metric does not account for maintenance considerations, availability is a more suitable metric to measure the effectiveness of an existing system. Furthermore, availability can be improved in an existing system through improved maintainability when the reliability values are not realized according to the manufacturer's specifications. $^{15}$

There are several definitions of availability depending on the user's point of view. All such definitions use equation (1) as a baseline, which essentially provides a probability of the system being operational. The various availability definitions differ in how they define what is included in the uptime and downtime parameters. The three most common categories of availability are inherent availability, achieved availability, and operational availability ${ }^{16}$

$$
\text { availability }=\frac{\text { uptime }}{\text { uptime }+ \text { downtime }}
$$

Inherent availability $\left(A_{i}\right)$ is the most commonly used availability measurement. Inherent availability uses the component's or system's mean time between failures (MTBF) as the uptime measure and mean time to repair (MTTR) as the downtime measure. MTTR includes only corrective maintenance (CM) downtime. The $A_{i}$ parameters initially come from the specifications in the manufacturer's report on how long the system is projected to operate before failure and how long it takes a normal maintenance team to repair a failed unit.

Operational availability $\left(A_{\mathrm{o}}\right)$ is the best measure of the "realistic" availability a user of a system actually experiences over a period of time. This is because $A_{\mathrm{o}}$ is based on the collection of all the actual events that occur to the system during any system downtime until the system is once again fully restored. It includes uptime as the mean time between maintenance 
(MTBM) actions and an additional term for ready time (RT), assuming the system is operational even if it is offline. The operational cycle is the total time being considered for the system. For the downtime parameter, $A_{\mathrm{o}}$ defines mean down time (MDT) with an expanded definition of the time to repair the system plus the delay time (DT). The significant aspect of the MDT is that it includes administrative and logistic delays, while the system is down, and the system's mean maintenance time $(\mathrm{M}) .^{16}$

Achieved availability $\left(A_{\mathrm{a}}\right)$ is the measure that the maintenance department would most often be tracking as a department performance measure of both the systems they maintain and the department capacity to maintain them, as it is based on both the actual maintenance touch time and the equipment's failure activity. The $A_{\mathrm{a}}$ definition and equation uses MTBM and M as its parameters. Achieved availability for a system with $n$ components is calculated in equation (2) ${ }^{16}$

$$
A_{\mathrm{a}}=\frac{\mathrm{MTBM}}{\mathrm{MTBM}+\mathrm{M}}
$$

$A_{\mathrm{a}}$ includes both CM actions, in the form of system failures, and PM actions that take the system offline, in the form of system downing PMs

$$
\begin{aligned}
& \text { MTBM }= \\
& \text { \# of system failures }+ \text { \# of system downing PMs } \\
& \mathrm{M}=\frac{\mathrm{CM} \text { downtime }+ \text { PM downtime }}{\# \text { of system failures }+ \text { \# of system downing PMs }}
\end{aligned}
$$

The weakness of $A_{i}$ is that it does not include the PM parameter incorporated into the calculation. The weakness of $A_{\mathrm{o}}$ is that it is convoluted with many other logistics and administrative parameters and delays. The conclusion is that neither of these first two availability calculations are well suited for focusing on maintenance impacts to availability. $A_{\mathrm{a}}$ is the most appropriate for this study as it incorporates PM and inspections can be split out of PM to analyze their core impact on $A_{\mathrm{a}}$.

\section{Importance measures}

It is very rare that systems are simplistic enough to have a minimum amount of components that would allow equal attention or worth to be given to all components. This is particularly true in the case of DoD weapons systems, which are often highly complex. Such systems lend themselves to allocate resources by dividing the system up into subsystems or collections of subsystems based on how important that subsystem is to the overall system. Then each subsystem can be more easily analyzed at the component level. The importance of each component to the subsystem can be measured and then the component's importance related to the overall aircraft.

Many CIMs have been developed to determine the criticality of individual components to system performance. ${ }^{17}$ Primarily, importance measures have been introduced to measure the influence of particular components on the overall reliability of the system. ${ }^{18,19}$ Specific CIMs include risk reduction worth (RRW), an index that quantifies the potential damage to a system caused by a particular component failure, and the reliability achievement worth (RAW) of a component, or the maximum proportion increase in system reliability generated by that component. ${ }^{20}$

This work will focus on the Birnbaum importance measure, among the most widely used importance measures in reliability engineering. ${ }^{21}$ For a system of $n$ components, the Birnbaum ${ }^{22}$ importance measure has historically measured how the change in reliability of component $i$ influences a change in the reliability of the system, or $I_{i}^{B}=\partial R_{s} / \partial R_{i}$. After the $I_{i}^{B}$ factor for each component is computed, the component with the largest $I_{i}^{B}$ value is the component that will offer the greatest improvement in system reliability when its reliability is improved.

Both Cassady et al. ${ }^{11}$ and Barabady and Kumar ${ }^{12}$ adapted the $I_{i}^{B}$ concept to propose an availability importance measure based on inherent availability. Their availability importance measures, in equation (5), demonstrates how the inherent availability of the system is influenced by the inherent availability of a subsystem or component. In their equations, $A_{s}$ is the system availability and $A_{i}$ is the component availability for component $i$

$$
I_{i}^{A}=\frac{\partial A_{s}}{\partial A_{i}}
$$

Similar to Birnbaum reliability importance, the change in the component availability that will have the greatest impact on the reliability of the system is from the $I_{i}^{A}$ with the largest value. This article also extends the Birnbaum reliability importance measures to availability, but in this article it is based on $A_{\mathrm{a}}$, considered to be a much more appropriate maintenance-centered availability measure. Furthermore, we focus on importance relative to MTBM and $\mathrm{M}$ measures.

\section{System configurations and their availability measures}

This article addresses the four primary system configurations depicted in Figure 1: series, parallel, series-parallel, and parallel-series. For these four system configurations, the notation for achieved availability is as follows: $A_{\mathrm{a}}^{\mathrm{S}}$ for series systems, $A_{\mathrm{a}}^{\mathrm{P}}$ for parallel systems, $A_{\mathrm{a}}^{\mathrm{SP}}$ for series-parallel, and $A_{\mathrm{a}}^{\mathrm{PS}}$ for parallel-series. For a series-only combination of components, the subscript $i$ represents any one of the $n$ individual components in the series system. For parallel configurations, the subscript $j$ refers to any one of the $m$ individual 


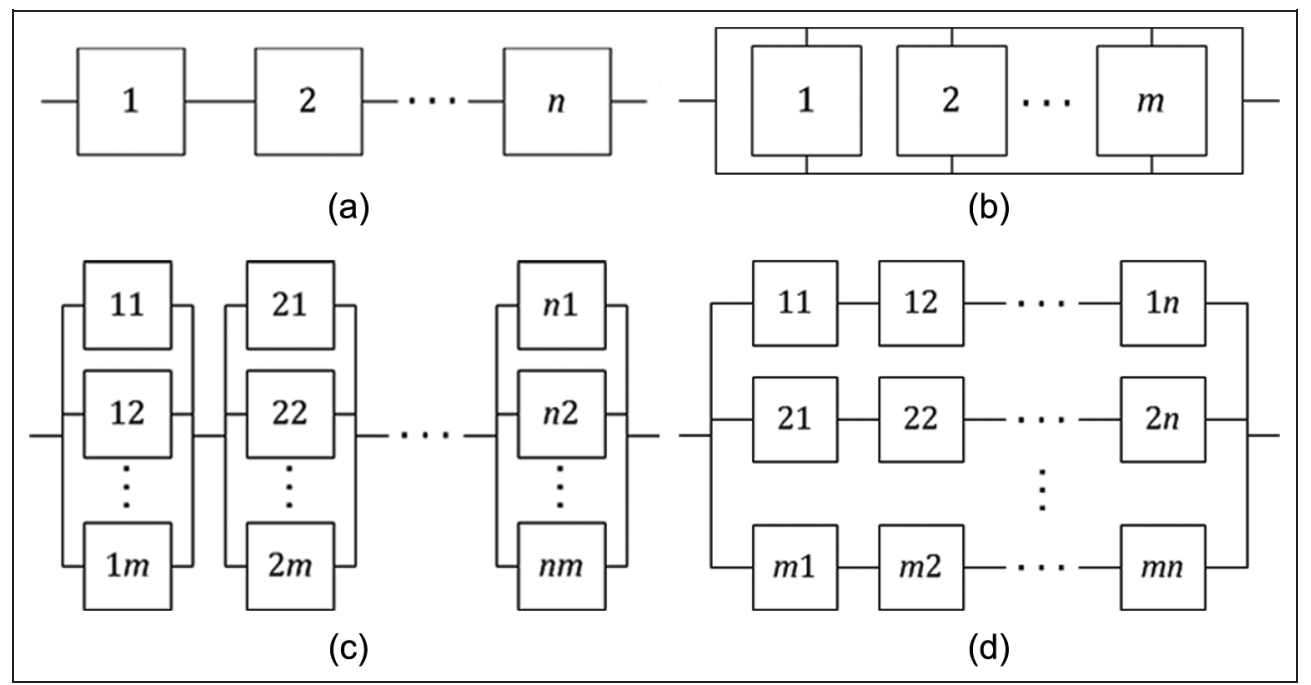

Figure I. Four primary configurations that describe the structure of most systems: (a) series, (b) parallel, (c) series-parallel, and (d) parallel-series.

parallel components of the parallel system or subsystem. For the series-parallel and parallel-series combination, the achieved availability notation includes either $i j$ or $j i$ to represent the individual components of the respective systems. The achieved availability calculations for particular system configurations are variants of the general form in equation (2).

Series systems. Figure 1(a) depicts a system comprised of $n$ independent components in series. For this system to be available, each independent component must be operable. The steady-state availability for a series system is the product of the independent component availabilities. Therefore, the system availability, $A_{\mathrm{a}}^{\mathrm{S}}$ found in equation (6), will be smaller than the smallest component's availability ${ }^{2}$

$$
A_{\mathrm{a}}^{\mathrm{S}}=\prod_{i=1}^{n} A_{\mathrm{a}_{i}}=\prod_{i=1}^{n} \frac{\mathrm{MTBM}_{i}}{\mathrm{MTBM}_{i}+\mathrm{M}_{i}}
$$

Parallel systems. Figure 1(b) depicts a system comprised of $m$ independent components connected in parallel. In order for this system to be unavailable, every independent component must be inoperable. This configuration can often be found when a system is critical to the operation of a weapons system (e.g. safety of flight for an aircraft). The $A_{\mathrm{a}}^{\mathrm{P}}$ is found in equation (7) as the probability that at least one component is not unavailable ${ }^{2}$

$$
\begin{aligned}
A_{\mathrm{a}}^{\mathrm{P}} & =\coprod_{j=1}^{m} A_{\mathrm{a}_{j}}=\coprod_{j=1}^{m} \frac{\mathrm{MTBM}_{j}}{\mathrm{MTBM}_{j}+\mathrm{M}_{j}} \\
& =1-\prod_{j=1}^{m}\left(1-\frac{\mathrm{MTBM}_{j}}{\mathrm{MTBM}_{j}+\mathrm{M}_{j}}\right)
\end{aligned}
$$

Series-parallel systems. A series-parallel system, depicted in Figure 1(c), is comprised of $n$ independent subsystems connected in series, where each subsystem consists of $m$ components in parallel. This is one of the more complicated systems but is also among the more common situations for a high-risk system (e.g. DoD aircraft). The redundancy allows some of the individual components to not be available and the system to still be available as long as at least one component in every subsystem is available. The achieved availability for this configuration is provided in equation (8)

$$
\begin{aligned}
A_{\mathrm{a}}^{\mathrm{SP}} & =\prod_{i=1}^{n}\left[\coprod_{j=1}^{m} A_{\mathrm{a}_{i j}}\right] \\
& =\prod_{i=1}^{n}\left[1-\prod_{j=1}^{m}\left(1-\frac{\mathrm{MTBM}_{i j}}{\mathrm{MTBM}_{i j}+\mathrm{M}_{i j}}\right)\right]
\end{aligned}
$$

Parallel-series systems. A parallel-series system, shown in Figure 1(d), is a system comprised of $m$ independent subsystems connected in parallel, where each subsystem consists of $n$ components in a series configuration. A parallel-series system configuration allows for multiple components to not be available, as long as all the components in one of the subsystems are still available. Such a system configuration is especially useful in describing the subsystems of an aircraft system, illustrated subsequently. The achieved availability in terms of the MTBM and M metrics of individual components is found in equation (9)

$$
\begin{aligned}
A_{\mathrm{a}}^{\mathrm{PS}} & =\coprod_{j=1}^{m}\left(\prod_{i=1}^{n} A_{\mathrm{a}_{j i}}\right) \\
& =1-\prod_{j=1}^{m}\left(1-\prod_{i=1}^{n} \frac{\mathrm{MTBM}_{j i}}{\mathrm{MTBM}_{j i}+\mathrm{M}_{j i}}\right)
\end{aligned}
$$




\section{Achieved availability importance measures}

Throughout this article, independence is assumed for all the components of a system, suggesting that the failure of one component does not have an effect on the other components. However, depending on the configuration of the system, the system may be either in a failed or working state. Assumptions for all systems are as follows, adapted from Cassady et al., ${ }^{11}$ and Barabady and Kumar: ${ }^{12}$

- The system is in steady state.

- Each system comprises components whose failures are independent.

- All components are repairable, which returns the component to an as good as new state.

- Each component and system has two states: working or down, where "down" includes inoperability due to failure and maintenance.

This section extends the Birnbaum importance measure with three importance measures, motivated by the approaches of Cassady et al. ${ }^{11}$ and Barabady and Kumar $^{12}$ but specifically (1) adapting achieved availability and (2) constructing two new improvement measures based on MTBM and M. The first importance measure is the achieved availability importance measure for the component and can be calculated when $i$ can equal $j, i j$, or $j i$, depending on the system configuration. $A_{\mathrm{a}}$ is the system-achieved availability and $A_{\mathrm{a}_{i}}$ is the $i$ th component's achieved availability, and the achieved availability importance measure $I_{\mathrm{a}_{i}}$ is found in equation(10)

$$
I_{\mathrm{a}_{i}}=\frac{\partial A_{\mathrm{a}}}{\partial A_{\mathrm{a}_{i}}}
$$

The other two importance measures are based on the availability of the system, with respect to the MTBM and $\mathrm{M}$ parameters. These two importance measures are referred to here as MTBM importance and $M$ importance.

The achieved availability importance measure with respect to the MTBM highlights how the MTBM, a surrogate measure of reliability, of component $i$ impacts the availability of the system. Provided in equation (11), the component with the largest value of $I_{\mathrm{a}, \mathrm{MTBM}_{i}}$ indicates that it has the largest effect on the availability for the system

$$
I_{\mathrm{a}, \mathrm{MTBM}_{i}}=\frac{\partial A_{\mathrm{a}}}{\partial \mathrm{MTBM}_{i}}=\frac{\partial A_{\mathrm{a}}}{\partial A_{\mathrm{a}_{i}}} \times \frac{\partial A_{\mathrm{a}_{i}}}{\partial \mathrm{MTBM}_{i}}
$$

The $\mathrm{M}$ importance measure highlights how the mean maintenance time of component $i$ impacts the availability of the entire system. The $\mathrm{M}$ of a component is a surrogate measure describing its maintainability. When evaluating equation (12), the $I_{\mathrm{a}, \mathrm{M}_{i}}$ resulting values are negative (as smaller values of $\mathbf{M}$ result in larger values of system availability). Since the goal of the importance analysis is to find the component that has the largest $I_{\mathrm{a}, \mathrm{M}_{i}}$ magnitude to determine the speed of which it changes, only the absolute values of the results of equation (12) are considered in the subsequent graphical comparisons of the values

$$
I_{\mathrm{a}, \mathrm{M}_{i}}=\frac{\partial A_{\mathrm{a}}}{\partial \mathrm{M}_{i}}=\frac{\partial A_{\mathrm{a}}}{\partial A_{\mathrm{a}_{i}}} \times \frac{\partial A_{\mathrm{a}_{i}}}{\partial \mathbf{M}_{i}}
$$

Explicitly highlighting the contributions of MTBM and $\mathrm{M}$ in equation (5) allows us to pinpoint which component of availability to concentrate on for the most important components: improve reliability (e.g. through supplier selection) or improve maintainability (e.g. through MRO on-hand inventory).

Characteristically, the MTBM is a much greater value than the $M$. When that is the case, $I_{a}, M_{i}$ will be much greater than $I_{\mathrm{a}, \mathrm{MTBM}_{i}}$ suggesting that finding ways to decrease the component's $M$ offers a greater benefit to the system availability than working on the component's MTBM. However, the cost and effort required to decrease $M$ may be more significant than what is required to increase the MTBM value. Depending on the MTBM and M values, the reverse situation could also be possible as well. To be certain the initial maintenance focus is on the correct parameter as determined by these additional importance measures, both the $I_{\mathrm{a}, \mathrm{MTBM}_{i}}$ and the $I_{\mathrm{a}, \mathrm{M}_{i}}$ should always be calculated.

\section{$A_{a}$ importance measures for a series system}

From equation (6), the achieved availability importance measure is found in equation (13) for a series system

$$
I_{\mathrm{a}_{i}}^{\mathrm{S}}=\frac{\partial A_{\mathrm{a}}^{\mathrm{S}}}{\partial A_{\mathrm{a}_{i}}}=\prod_{k \neq i} A_{\mathrm{a}_{k}}
$$

The MTBM importance and M importance are computed for the target component in the series system, shown in equations (14) and (15), respectively

$$
\begin{aligned}
& I_{\mathrm{a}, \mathrm{M}_{i}}^{\mathrm{S}}=I_{\mathrm{a}_{i}}^{\mathrm{S}} \times \frac{\partial A_{\mathrm{a}_{i}}}{\partial \mathrm{M}_{i}}=\frac{\partial A_{\mathrm{a}}^{\mathrm{S}}}{\partial A_{\mathrm{a}_{i}}} \times \frac{\partial A_{\mathrm{a}_{i}}}{\partial \mathrm{M}_{i}} A_{\mathrm{a}}^{\mathrm{S}} \times \frac{1}{\left(\mathrm{MTBM}_{i}+\mathrm{M}_{i}\right)} \\
& I_{\mathrm{a}, \mathrm{MTBM}_{i}}^{\mathrm{S}}=\frac{\partial A_{\mathrm{a}}^{\mathrm{S}}}{\partial A_{\mathrm{a}_{i}}} \times \frac{\partial A_{\mathrm{a}_{i}}}{\partial \mathrm{MTBM}_{i}}=I_{\mathrm{a}_{i}}^{\mathrm{S}} \times \frac{\partial A_{\mathrm{a}_{i}}}{\partial \mathrm{MTBM}_{i}} \\
&=A_{\mathrm{a}}^{\mathrm{S}} \times \frac{\mathrm{M}_{i}}{\operatorname{MTBM}_{i}\left(\mathrm{MTBM}_{i}+\mathrm{M}_{i}\right)}
\end{aligned}
$$

\section{$A_{a}$ importance measures for a parallel system}

From the definition of parallel system-achieved availability in equation (7), the associated importance measure, $I_{\mathrm{a}_{j}}^{\mathrm{P}}$, is provided in equation (16)

$$
I_{\mathrm{a}_{j}}^{\mathrm{P}}=\frac{\partial A_{\mathrm{a}}^{\mathrm{P}}}{\partial A_{\mathrm{a}_{j}}}=1-\prod_{l \neq j}\left(1-A_{\mathrm{a}, l}\right)
$$


With equations (17) and (18), respectively, the MTBM and M importance measures can be further computed for the target component in a parallel system

$$
\begin{aligned}
I_{\mathrm{a}, \mathrm{MTBM}_{j}}^{\mathrm{P}}= & \frac{\partial A_{\mathrm{a}}^{\mathrm{P}}}{\partial A_{\mathrm{a}_{j}}} \times \frac{\partial A_{\mathrm{a}_{j}}}{\partial \mathrm{MTBM}_{j}} \\
= & {\left[1-\prod_{l \neq j}\left(1-A_{\mathrm{a}_{l}}\right)\right] } \\
& \times \frac{\mathrm{M}_{j}}{\mathrm{MTBM}_{j}\left(\mathrm{MTBM}_{j}+\mathrm{M}_{j}\right)} \times A_{\mathrm{a}_{j}} \\
I_{\mathrm{a}, \mathrm{M}_{j}}^{\mathrm{P}}= & \frac{\partial A_{\mathrm{a}}^{\mathrm{P}}}{\partial A_{\mathrm{a}_{j}}} \times \frac{\partial A_{\mathrm{a}_{j}}}{\partial \mathrm{M}_{j}}=\left[1-\prod_{l \neq j}\left(1-A_{\mathrm{a}_{l}}\right)\right] \\
\times & \frac{1}{\left(\mathrm{MTBM}_{j}+\mathrm{M}_{j}\right)} \times A_{\mathrm{a}_{j}}
\end{aligned}
$$

\section{$A_{a}$ importance measures for a series-parallel system}

Equation (19) illustrates the impact of the achieved availability $i j$ th component on the system-achieved availability for a series-parallel system. Priority of ranking of which component to start with, in terms of best choice for increasing the system availability, should be assigned to component $i j$ with the maximum $I_{\mathrm{a}_{i j}}^{\mathrm{SP}}$. Focus is given to the impact of MTBM and M to systemachieved availability with equations (20) and (21)

$$
\begin{array}{r}
I_{\mathrm{a}_{i j}}^{\mathrm{SP}}=\frac{\partial A_{\mathrm{a}}^{\mathrm{SP}}}{\partial A_{\mathrm{a}_{i j}}}=\prod_{k \neq i}\left[1-\prod_{l=1}^{m}\left(1-A_{\left.\mathrm{a}_{k l}\right)}\right) \times \prod_{l \neq j}\left(1-A_{\mathrm{a}_{i}}\right)\right. \\
I_{\mathrm{a}, \mathrm{MTBM}_{i j}}^{\mathrm{SP}}=\frac{\partial A_{\mathrm{a}}^{\mathrm{SP}}}{\partial A_{\mathrm{a}_{i j}}} \times \frac{\partial A_{\mathrm{a}_{i j}}}{\partial \mathrm{MTBM}_{i j}} \\
=I_{\mathrm{a}_{i j}}^{\mathrm{SP}} \times \frac{\mathrm{M}_{i j}}{\mathrm{MTBM}_{i j}\left(\mathrm{MTBM}_{i j}+\mathrm{M}_{i j}\right)} \times A_{\mathrm{a}_{i j}} \\
I_{\mathrm{a}, \mathrm{M}_{i j}}^{\mathrm{SP}}=\frac{\partial A_{\mathrm{a}}^{\mathrm{SP}}}{\partial A_{\mathrm{a}_{i j}}} \times \frac{\partial A_{\mathrm{a}_{i j}}}{\partial \mathrm{M}_{i j}}=I_{\mathrm{a}_{i j}}^{\mathrm{SP}} \times \frac{1}{\left(\mathrm{MTBM}_{i j}+\mathrm{M}_{i j}\right)} \times A_{\mathrm{a}_{i j}}
\end{array}
$$

\section{$A_{a}$ importance measures for a parallel-series system}

From the achieved availability calculation for a parallel-series system in equation (9), the importance measure for component $j i$ achieved availability provided in equation (22) is used to compute the achieved availability importance measures for a parallel-series system

$$
I_{\mathrm{a}_{j i}}^{\mathrm{PS}}=\frac{\partial A_{\mathrm{a}}^{\mathrm{PS}}}{\partial A_{\mathrm{a}_{j i}}}=\prod_{l \neq i}\left[1-\prod_{k=j}^{n} A_{\mathrm{a}_{l k}}\right] \times \prod_{k \neq j} A_{\mathrm{a}_{j k}}
$$

The MTBM and M importance measures are computed for the target component in a parallel-series system in equations (23) and (24)

$$
\begin{aligned}
& I_{\mathrm{a}, \mathrm{MTBM}_{j i}}^{\mathrm{PS}}=\frac{\partial A_{\mathrm{a}}^{\mathrm{PS}}}{\partial A_{\mathrm{a}_{j i}}} \times \frac{\partial A_{\mathrm{a}_{j i}}}{\partial \mathrm{MTBM}_{j i}} \\
&=I_{\mathrm{a}_{j i}}^{\mathrm{PS}} \times \frac{\mathrm{M}_{j i}}{\mathrm{MTBM}_{j i}\left(\mathrm{MTBM}_{j i}+\mathrm{M}_{j i}\right)} \times A_{\mathrm{a}_{j i}} \\
& I_{\mathrm{a}, \mathrm{M}_{j i}}^{\mathrm{PS}}=\frac{\partial A_{\mathrm{a}}^{\mathrm{PS}}}{\partial A_{\mathrm{a}_{j i}}} \times \frac{\partial A_{\mathrm{a}_{j i}}}{\partial \mathrm{M}_{j i}}=I_{\mathrm{a}_{j i}}^{\mathrm{PS}} \times \frac{1}{\left(\mathrm{MTBM}_{j i}+\mathrm{M}_{j i}\right)} \times A_{\mathrm{a}_{\mathrm{a} j i}}
\end{aligned}
$$

\section{Illustrative examples: modeling the impact of inspections}

An inspection decision-making framework motivated by the achieved availability importance measures is provided in this section, wherein we identify the components that have the largest magnitude importance measure and also maximize the system-achieved availability for each system by allowing the adjustment of the number of inspections for each component. The four system configurations are illustrated with an example inspired by the components of an aircraft.

\section{$A_{a}$ importance measure decision-making framework}

Often MTBM and M measures do not differentiate between PM actions (the physical activity required to performance PM) and inspection actions (which may or may not result in PM actions depending on the result of the inspection). As a primary objective of this IM-inspired framework is to assist in determining the frequency of inspections, the difference between PM and inspection should be explicit. As such, the calculations of MTBM in equation (3) and M in equation (4) are modified with equations (25) and (26) by separating out the parameter "\# of Inspections" in the denominators of both equations and the parameter "Inspection Time" in the numerator of equation (26). Note that the effect of decreasing the number of inspections in equations (25) and (26) is an increase the inspection interval

$$
\mathrm{MTBM}=\frac{\text { Total uptime }}{\# \text { of CMs }+\# \text { of PMs }+\# \text { of Inspections }}
$$

$$
\mathrm{M}=\frac{\mathrm{CM} \text { downtime }+ \text { PM downtime }+ \text { Inspection time }}{\# \text { of CMs }+\# \text { of PMs }+\# \text { of Inspections }}
$$

Recall the basic availability formula in equation (1), the ratio of uptime to uptime + downtime. To maximize availability, either uptime has to be maximized or downtime has to be minimized. Downtime, the numerator in equation (26), can be expressed with equation (27), where $n_{C M}, n_{P M}$, and $n_{I}$ refer to the numbers of $\mathrm{CM}, \mathrm{PM}$, and inspection actions, respectively, and $y_{C M}, y_{P M}$, and $y_{I}$ refer to the mean downtime for those actions. The total downtime for $\mathrm{CM}$ is $n_{C M} y_{C M}$, the total downtime for PM is $n_{P M} y_{P M}$, and the total downtime for the inspection is $n_{I} y_{I}$ 
downtime $=n_{\mathrm{CM}} y_{\mathrm{CM}}+n_{\mathrm{PM}} y_{\mathrm{PM}}+n_{\mathrm{I}} y_{\mathrm{I}}$

For those components deemed important to availability by the importance measures above, particularly those important to the mean downtime metric, $\mathrm{M}$, the optimal number of inspections to improve availability is determined with the conceptual optimization problem found in equation (28) for component $i$. To accommodate for inspection-related decisions, the CM downtime and PM downtime calculations are modified. Two new parameters are introduced: $\mathrm{P}$ is the number of inspections before a replacement is done, and $\mathrm{C}$ is a proportional multiplier to be applied to the repair time to determine the replacement time. In addition, revisions from equation (27) include these new two new parameters to account for inspection-related decisions. CM downtime is impacted when an inspection action occurs by avoiding a failure and instead doing a replacement, indicating a modification to the time associated with a replacement instead of a repair. Furthermore, a repair time for $\mathrm{CM}$ is avoided, thus adding an additional modification to the equation. Finally, a reduction of one PM action occurs when avoiding a failure. In typical maintenance environments, all variables would be set values for equation (28) and assumed for a steady-state system

$$
\begin{aligned}
& \min _{n_{I_{i}}}\left(n_{\mathrm{PM}_{i}}-\frac{n_{\mathrm{I}_{i}}}{P}\right) y_{\mathrm{CM}_{i}}+\left(C y_{\mathrm{CM}_{i}}-\frac{n_{\mathrm{I}_{i}}}{P}\right) \\
& +\left(n_{\mathrm{PM}_{i}}-\frac{n_{\mathrm{I}_{i}}}{P}\right) y_{\mathrm{PM}_{i}}+\left(n_{I_{i}} y_{\mathrm{I}_{i}}\right) \text { s.t. } n_{\mathrm{I}_{i}} \geqslant 0
\end{aligned}
$$

all other parameters $n_{\mathrm{CM}_{i}}, n_{\mathrm{PM}_{i}}, y_{\mathrm{CM}_{i}}, y_{\mathrm{PM}_{i}}, y_{\mathrm{I}_{i}} \geqslant 0$, $0 \leqslant C \leqslant 1,0 \leqslant P \leqslant 1$.

The ranking of components according to their availability importance measures produces a lexicographically ordered maximum value set, of which $i$ is a member. The optimization problem in equation (28) could be performed for multiple components in this set, or an optimization problem could be designed to account for the inspection of multiple components simultaneously if applicable.

\section{Illustrative example background: aircraft maintenance}

Field aircraft maintenance data were acquired to illustrate the application of all the different example systems by applying the data in the decision-making framework for the different achieved availability importance measures to each appropriate system. Note that a random factor was applied to the raw data to mask the aircraft from which the data were derived and also the component names are not used. However, proportional relationships among components and the overall performance parameters are still reasonably characteristic in the resulting component priority predictions made by the CIM determinations.

The data were collected for a parallel-series system that comprises a parallel system made up of four

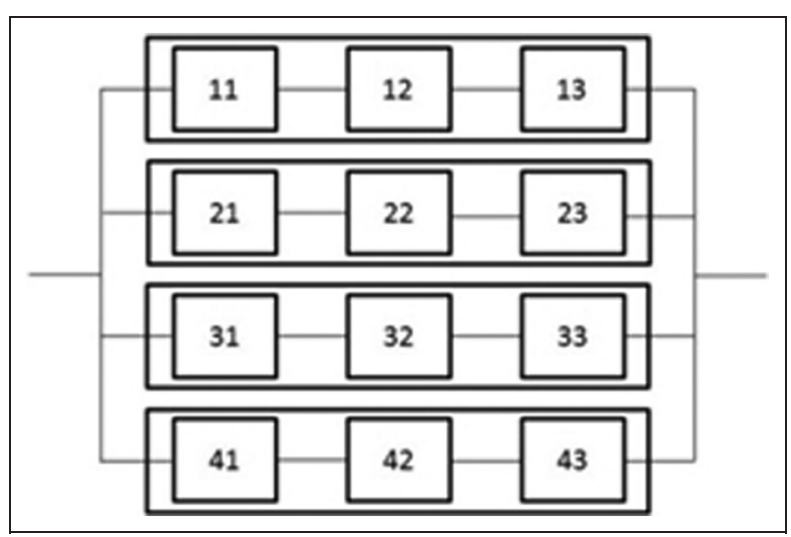

Figure 2. Diagram of the parallel-series aircraft system configuration.

subsystems, with each subsystem a series configuration with three components. The individual component names are referred to as Component 11, Component $12, \ldots$, Component $j i$ because the actual configuration is in parallel-series. This scenario is depicted in Figure 2.

Data were collected for a 1-year period (potential maximum total uptime time of $8760 \mathrm{~h}$ ) from field aircraft and are used for the illustration and analysis sections of this article. The data representing the variables in equation (28), shown in Table 1, include the number of and downtime length for $\mathrm{CM}$ actions, the number of and downtime length for PM actions, and the number of and downtime length for inspections.

The inspection that provides the insight to replace the component before it fails is called the "critical" inspection. The actual number of inspections needed before the critical inspection occurs would be determined by the historical inspection data and communicating with maintenance analysts. It is assumed that the number of inspections added before the critical inspection yields an accurate failure prediction is a fully adjustable parameter for each component.

The amount of time that a replacement takes is also a parameter in the decision-making framework to allow variation in different component experiences. When a component is replaced, as determined by the critical inspection, the mean replacement time is used in place of the mean failure time and the CM and PM counts are reduced by one event. This assumption is realistic because after the replacement of a component, CM, and PM would not be performed on the brand new equipment.

Subsets of this aircraft illustrative example will be used to illustrate a series subsystem configuration and the actual parallel-series configuration of Figure 2.

\section{$A_{a}$ framework example: series system}

The data chosen from Table 1 for the series system describe Components 11, 12, and 13, comprising the 
Table I. CM, PM, and inspection data for the aircraft system (time measured in hours).

\begin{tabular}{|c|c|c|c|c|c|c|}
\hline Component & No. of CM down & Time per CM & No. of PM down & Time per PM & No. of inspections & Time per inspection \\
\hline$j i$ & $n_{\mathrm{CM}}$ & YСM & $n_{\mathrm{PM}}$ & $y_{\mathrm{PM}}$ & $n_{1}$ & $y_{1}$ \\
\hline II & 10 & 1.59 & 46 & 3.66 & 7 & 9.78 \\
\hline 12 & 12 & $|3.3|$ & 41 & 4.03 & 7 & 9.78 \\
\hline 13 & 19 & 2.81 & 53 & 4.09 & 7 & 9.78 \\
\hline 21 & 22 & 2.88 & 60 & 5.58 & 9 & 8.16 \\
\hline 22 & 15 & 4.63 & 80 & 6.76 & 5 & $\mathrm{I} .47$ \\
\hline 23 & 20 & 5.27 & 66 & 9.67 & 5 & 1.70 \\
\hline 31 & 20 & 5.74 & 75 & 6.60 & 7 & 3.95 \\
\hline 32 & 10 & 5.10 & 73 & 5.94 & 7 & 2.13 \\
\hline 33 & 15 & 43.01 & 29 & 15.13 & 3 & 24.14 \\
\hline 41 & 10 & 37.74 & 19 & I5.04 & 3 & 8.93 \\
\hline 42 & 14 & 47.47 & 26 & 14.59 & 5 & 19.04 \\
\hline 43 & 10 & 53.47 & 19 & 16.91 & 3 & 24.14 \\
\hline
\end{tabular}

CM: corrective maintenance; PM: preventive maintenance.

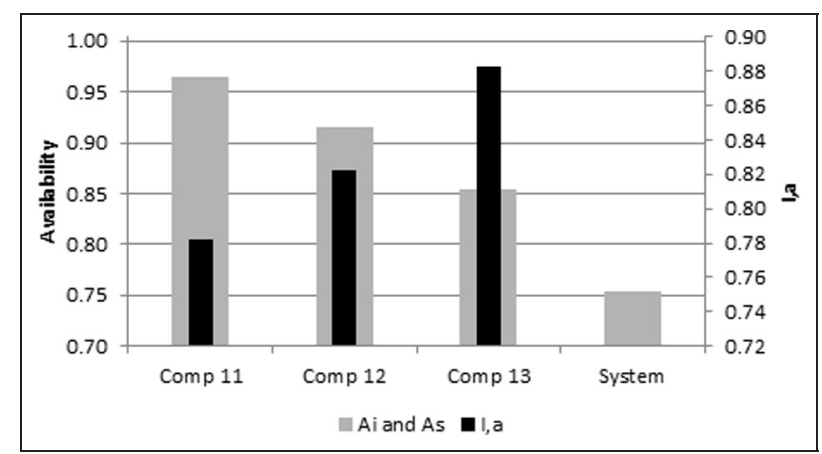

Figure 3. Availability and $A_{a}$ importance for components in the series subsystem.

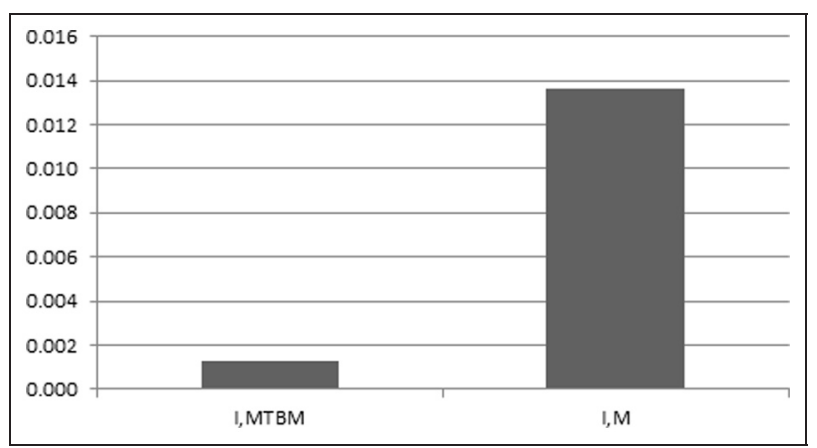

Figure 4. Comparing MTBM and M importance for Component 13.

MTBM: mean time between maintenance; M: mean maintenance time.

first of the four series subsystem. Figure 3 depicts each of the individual component availabilities and the system availability, all shaded in gray. The $I_{\mathrm{a}_{i}}^{\mathrm{S}}$ for each component is shown in black. Note the different vertical axes: the trend of the relationship between the two calculations is of greater interest. As expected for a series system, the component with the least availability is the component with the largest system importance measures. From the calculation of the importance measures, it is seen that Component 13 becomes the clear

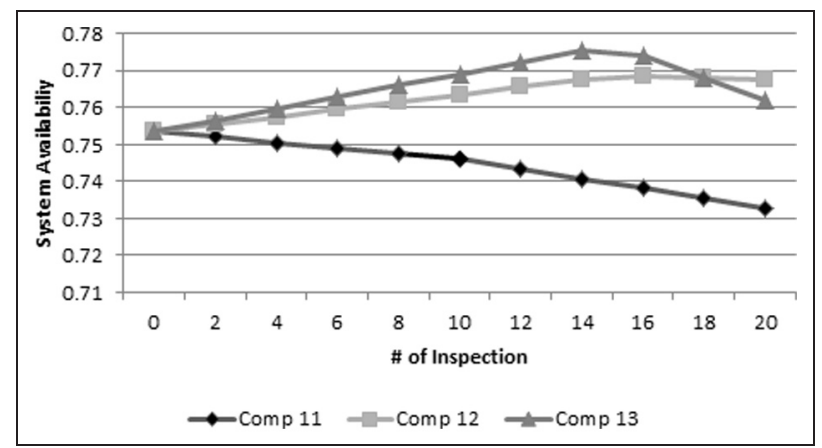

Figure 5. Series subsystem $A_{\mathrm{a}}$ sensitivity analysis.

choice with the highest priority component to focus improvement efforts: the more available Component 13 becomes, the more available the system becomes.

Now that Component 13 has been identified, the next step is to make a comparison between $I_{\text {a, }}^{S}$ MTBM $_{i}$ and $I_{\mathrm{a}, \mathrm{M}_{i}}^{\mathrm{S}}$ for Component 13 to further determine which of these parameters can provide the greatest positive impact on the system availability. This comparison is shown in Figure 4, which depicts that the $I_{\mathrm{a}, \mathrm{M}_{i}}^{\mathrm{S}}$ is greater compared to $I_{\mathrm{a}, \mathrm{MTBM}_{i}}^{\mathrm{S}}$. This suggests that improving M, or improving the maintainability of the component, for Component 13 provides most improvement for system availability.

Given that Component 13 and the $\mathrm{M}$ parameter have been identified as having most impact on system availability, equation (28) is deployed in a discrete form to determine the number of inspections for each component (taken individually) to further influence system availability. Figure 5 provides something of a sensitivity analysis, varying each component individually while increasing the number of inspections to visualize the impact to the system availability. Figure 5 shows that, indeed, Component 13 has the fastest and greatest impact on the system. When the inspections are increased for Component 13, it provides the greatest opportunity to increase system availability up to 14 inspections. After that, the downtime attributed to 


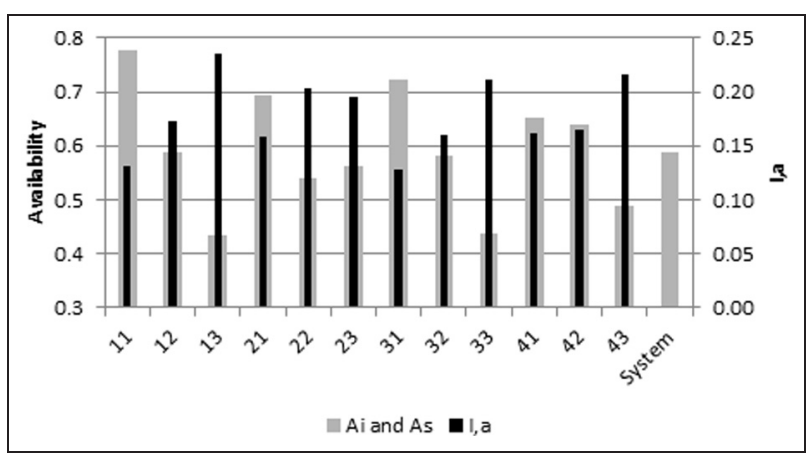

Figure 6. Availability and $A_{a}$ importance for components in the entire parallel-series system.

inspections outweighs the downtime due to potential $\mathrm{CM}$ actions.

Notice, Component 11 actually shows a negative impact on the system availability while increasing inspections. This is due to the inspection time for Component 11 being significantly longer than the replace or repair time. This points out an area for a maintenance process improvement, as an inspection likely should not take such an amount of time that the system cannot benefit from an inspection.

All these steps and analysis can now be repeated for the remainder of the system configurations. What was intuitively predictable for a series system without the use of importance measure computations becomes untenable for the more complex configurations, only with the computations, charts, and graphs does it become immediately possible to make the same type of results and optimal predictions for the systems.

\section{$A_{a}$ framework example: parallel-series system}

A series-parallel illustration is not provided, as the example in Figure 2 does not readily lend itself to such an application. Focus is given to the actual configuration in Figure 2, the parallel-series system, which is not featured in the work of Barabady and Kumar. ${ }^{12}$ No changes to the structure of the data in Table 1 were made for this illustration.

Figure 6 shows the individual component availabilities and system availability in gray, with the $I_{\mathrm{a}_{j i}}^{\mathrm{PS}}$ measures for each component in black. The component with the largest $I_{\mathrm{a}_{i i}}^{\mathrm{PS}}$ is shown as Component 13, which is prioritized as the first to focus improvement efforts. However, there are other components with $I_{\mathrm{a}_{i j}}^{\mathrm{PS}}$ values that are nearly the same magnitude and could also be under consideration for improvement, including Components 43 and 33. These components should be ranked in priority, and other factors (e.g. budget, manpower, space constraints) not chosen in this analysis could change the lexicographic order of these highly ranked components. The following analysis will focus on the highest ranked, Component 13.

A comparison for Component 13 between $I_{\mathrm{a}, \mathrm{MTBM}}^{\mathrm{PS}}$ and $I_{\mathrm{a}, \mathrm{M}_{j i}}^{\mathrm{PS}}$ further determines which parameter can have

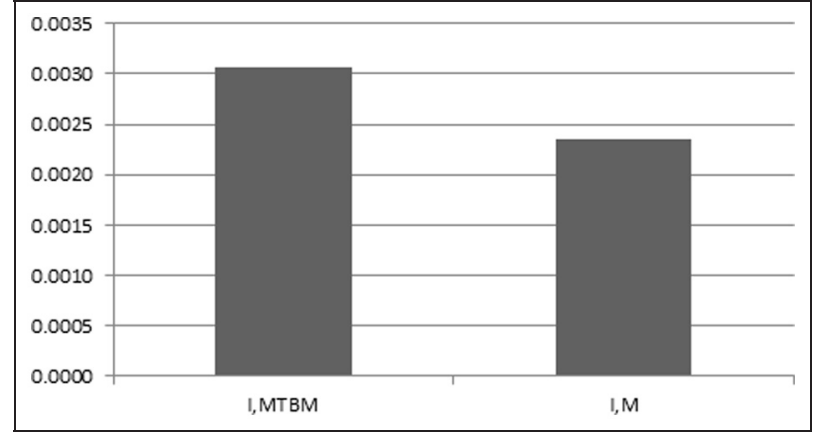

Figure 7. Comparing MTBM and $M$ importance for Component 13.

MTBM: mean time between maintenance; M: mean maintenance time.

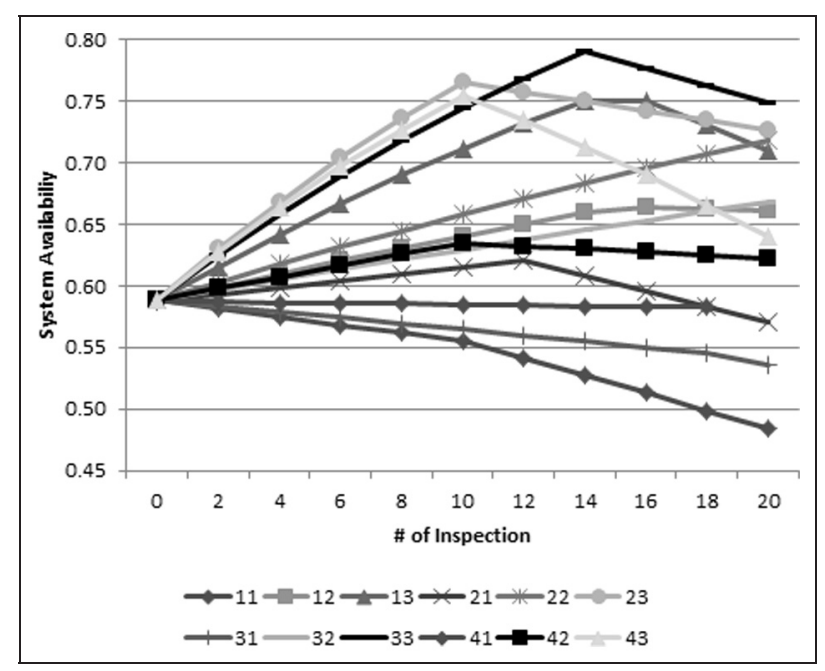

Figure 8. Parallel-series system $A_{a}$ sensitivity analysis.

the greatest impact on the system. This comparison is done in the chart in Figure 7, which suggests Component 13 shows the $I_{\mathrm{a}, \mathrm{MTBM}_{i i}}^{\mathrm{PS}}$ is the one with the larger magnitude. Thus, an improvement on the MTBM for Component 13 would be expected to yield a greater impact on the system availability with the less effort. This implies that the system is down more than it is up. Consider looking into a potential issue with the reliability causing extremely high failures or the maintenance techniques are taking an excessive amount of time.

Figure 8 provides the sensitivity implementation of equation (28) examining each component individually while increasing the inspections to see the impact to system availability.

Figure 8 shows that Component 33 reaches the highest system-achieved availability with critical inspections at inspection 14. Component 23 is the second highest, but takes fewer added inspections to reach its maximum impact to the system. Figure 8 indicates the third component for each subsystem (Components 13, 23, 33, and 43) as the components that can impact the systemachieved availability the greatest with critical 
inspections added. This has implications for the particular aircraft example.

\section{Concluding remarks}

This article presents a means to (1) extend existing availability importance measures to include the effects of maintenance activity, (2) determine the number of inspections to obtain the maximum system-achieved availability, and (3) apply this new availability importance measure tied to the optimal inspection frequency within an RCM decision-making framework.

The availability-based framework has proven to be very useful to allow data to drive decisions when improving the achieved availability of a sample system. The importance measure is a deterministic objective measure that reports the status of the component, in particular, using achieved availability as its basis measure. It then points to the component that has the most opportunity for improvement, further identifying whether the MTBM or M parameter needs the most work for the system availability to improve. Each result is dependent on the status of all the components in the system and identifies directly the one that needs improvement. Otherwise, time and money can easily be wasted by putting efforts into something that could result in a reduced system-achieved availability.

An ideal realm to execute the findings of the decision-making framework is RCM. The main objective of RCM is to be used for determining optimal failure management tactics, to include maintenance policies and provide proof for results from maintenance tasks. $^{8}$ The illustrative decision-making framework results assist by allowing data to efficiently and effectively drive decisions to accomplish the RCM objective.

Further work lies in applying a simulation-based approach to determining the availability-based importance of the components in more complex structures, paralleling the analytical approach in this article.

\section{Acknowledgement}

The views expressed in written materials or publications do not necessarily reflect the official policies of the Naval Postgraduate School or US Air Force nor does mention of trade names, commercial practices, or organizations imply endorsement by the US Government.

\section{Declaration of conflicting interests}

The authors declare that there is no conflict of interest.

\section{Funding}

This publication results from research supported by the Naval Postgraduate School Assistance Grant/ Agreement No. N00244-14-1-0027 awarded by the NAVSUP Fleet Logistics Center San Diego. Furthermore, this work was supported, in part, by tuition assistance funding provided by the US Air Force.

\section{References}

1. Department of Defense. Defense acquisition system DoD guide for achieving reliability, availability, and maintainability. Office of the Undersecretary of Defense, US Department of Defense, Washington, DC, August 2005.

2. Ebeling C. An introduction to reliability and maintainability engineering. Long Grove, IL: Waveland Press, Inc., 2010 .

3. Government Accountability Office. DOD needs to determine its aerial refueling aircraft requirements. GAO-04349, 4 June 2004. Washington, DC: Government Accountability Office.

4. Mathaisel DFX. Sustaining the military enterprise: an architecture for a lean transformation. New York: Auerbach Publications, 2008.

5. Kuo W and Prasad V. Reliability optimization of coherent systems. IEEE T Reliab 2000; 49(3): 323-330.

6. Misra KB and Sharma JJ. A new geometric programming formulation for a reliability problem. Int $J$ Control 1973; 18(3): 497-503.

7. Lie $\mathrm{CH}$ and Chun $\mathrm{YH}$. An algorithm for preventive maintenance policy. IEEE T Reliab 1986; 35(1): 71-75.

8. Department of Defense. Reliability centered maintenance manual. DoDM 4151.22-M, 30 June 2011. Washington, DC: US Department of Defense.

9. Government Accountability Office. The department needs a focused effort to overcome critical spare parts shortages. GAO-06-391, June 27, 2003. Washington, DC: Government Accountability Office.

10. Kuo $\mathrm{W}$ and Zhu $\mathrm{X}$. Importance measures in reliability, risk, and optimization: principles and applications. Chichester: Wiley, 2012.

11. Cassady CR, Pohl EA and Jin S. Managing availability improvement efforts with importance measures and optimization. IMA J Manag Math 2004; 15(2): 161-174.

12. Barabady JJ and Kumar UU. Availability allocation through importance measures. Int J Qual Reliab Manag 2007; 24(6): 643-657.

13. Moubray J. Reliability-centered maintenance. 2nd ed.New York: Industrial Press, Inc., 1997.

14. Endrenyi JJ, Aboresheid SS, Allan RN, et al. The present status of maintenance strategies and the impact of maintenance on reliability. IEEE T Power Syst 2001; 16(4): 638-646.

15. Kuo W and Wan R. Recent advances in optimal reliability allocation. IEEE T Syst Man Cy A 2006; 37(2): 143156.

16. Lie $\mathrm{CH}$, Hwang $\mathrm{CL}$ and Tillman FA. Availability of maintained systems: a state-of-the-art survey. AIIE T 1977; 9(3): 247-259.

17. Miman $M$ and Pohl EA. Uncertainty assessment for availability: importance measures. In: Proceedings of the IEEE reliability and maintainability symposium, Newport Beach, CA, 23-26 January 2006, pp.222-227. New York: IEEE.

18. Kuo W and Zuo MJ. Optimal reliability modeling: principles and applications. Hoboken, NJ: Wiley, 2003.

19. Modarres M, Kaminskiy M and Krivtsov V. Reliability engineering and risk analysis: a practical guide. 2nd ed.Boca Raton, FL: CRC Press, 2010.

20. Ramirez-Marquez JE, Rocco CM, Gebre BA, et al. New insights on multi-state component criticality and importance. Reliab Eng Syst Safe 2006; 91(8): 894-904. 
21. Fricks RM and Trivedi KS. Importance analysis with Markov chains. In: Proceedings of the annual reliability and maintainability symposium, Tampa, FL, 27-30 January 2003, pp.89-95. New York: IEEE.
22. Birnbaum ZW. On the importance of different component in a multi-component system. In: Krishnaiah (ed.) Multivariate analysis, vol. 11. New York: Academic Press, 1969. 